\title{
Norois
}

Environnement, aménagement, société

\section{Apport de l'approche systémique et pluridisciplinaire dans l'étude du domaine littoral et marin de la Seine-Maritime (France)}

Contribution of systemic and pluridisciplinary approach for the coastal and marine area of the Seine-Maritime (France)

Stéphane Costa, Françoise Gourmelon, Claude Augris, Philippe Clabaut et Bernard Latteux

\section{OpenEdition}

\section{Journals}

Édition électronique

URL : http://journals.openedition.org/norois/534

DOI : $10.4000 /$ norois. 534

ISBN : 978-2-7535-1544-4

ISSN : $1760-8546$

Éditeur

Presses universitaires de Rennes

Édition imprimée

Date de publication : 1 septembre 2005

Pagination : 91-108

ISBN : 978-2-7535-0218-5

ISSN : 0029-182X

Référence électronique

Stéphane Costa, Françoise Gourmelon, Claude Augris, Philippe Clabaut et Bernard Latteux, « Apport de l'approche systémique et pluridisciplinaire dans l'étude du domaine littoral et marin de la SeineMaritime (France) », Norois [En ligne], 196 | 2005/3, mis en ligne le 08 août 2008, consulté le 19 avril 2019. URL : http://journals.openedition.org/norois/534 ; DOI : 10.4000/norois.534

Ce document a été généré automatiquement le 19 avril 2019

(c) Tous droits réservés 


\section{Apport de l'approche systémique et pluridisciplinaire dans l'étude du domaine littoral et marin de la Seine-Maritime (France)}

Contribution of systemic and pluridisciplinary approach for the coastal and marine area of the Seine-Maritime (France)

Stéphane Costa, Françoise Gourmelon, Claude Augris, Philippe Clabaut et Bernard Latteux

\section{NOTE DE L'ÉDITEUR}

Cet article a été reçu le 14 juin 2005 et définitivement accepté le 18 octobre 2005.

1 Pour comprendre et gérer les littoraux, ceux-ci doivent être considérés comme un système ouvert dont l'équilibre dynamique est régi par des transferts constants d'énergie et de sédiments. La variation de ces flux, dans le temps et l'espace, conditionne l'état du stock sédimentaire mobilisable, et donc le rythme d'évolution morphologique du littoral. Cette approche morphodynamique, initiée dès le début des années 1970 (May et Tanner, 1973 ; Wright et Thom, 1977), nécessite de définir les limites spatiales du système qui n'ont rien de commun avec des frontières administratives trop restrictives. S'agissant des formes d'accumulation, notamment sableuses, il est communément admis l'existence d'interrelations entre les plages sub-aérienne et sous-marine qui conditionnent l'équilibre dynamique du rivage (Komar, 1983 ; Hess et Harris, 1987 ; Levoy, 1994 ; Hill et al., 2004).

Les côtes à falaises et les plages graveleuses, telles que celles du littoral haut-normand, sont également considérées comme un système puisque le recul des abrupts induit l'élargissement des plate-formes d'érosion marine, et alimente en silex le cordon de 
galets. En retour, ces deux modelés contribuent à réduire l'intensité et le temps d'action des vagues au pied des abrupts crayeux et donc leur instabilité. En revanche, pour ce type de côte, les échanges sédimentaires avec le large sont très rarement pris en compte en raison de la faible mobilité transversale des galets. En effet, le fonctionnement morphosédimentaire de ces plages, considérées uniquement comme des formations de haut estran, n'est envisagé que dans ses relations avec le recul des falaises ou bien sous l'angle de la mobilité de ces éléments constitutifs principaux, à savoir, la classe granulométrique dite des cailloutis $(>2 \mathrm{~cm})$. Or, ces estrans peuvent être également caractérisés par l'existence d'une fraction sableuse non négligeable en volume et en surface. Celle-ci se retrouve dans les cordons de galets (Kirk, 1980; Carter et Orford, 1984 ; Shulmeister et Kirk, 1993; Orford et al., 2002), et sur les platiers rocheux, dont certains, en HauteNormandie, ont connu, depuis le début des années 1990, un ensablement soudain et massif. Ces ensablements semblent influencer le fonctionnement morpho-sédimentaire des accumulations graveleuses (Costa, 1997, 1999; Costa et Delahaye, 2002, 2005). Ce constat soulève la question de l'origine, notamment infratidale, de ces matériaux dont les caractéristiques granulométriques sont similaires aux sables des petits fonds se trouvant dans la bande des 0 à $15 \mathrm{~m}$ (les profondeurs sont données en cote marine indiquée CM dans le texte).

L'objectif de cette contribution est donc de s'interroger sur la pertinence d'élargir vers le large les limites du système falaise/platier rocheux/cordon de galets haut-normand. Cette approche systémique, et nécessairement pluridisciplinaire, a été entreprise dans le cadre d'une étude qui a rassemblé des compétences en géographie, géologie, et en ingénierie (hydrodynamisme). Elle est fondée sur l'acquisition et l'analyse de données morpho-sédimentaires et bathymétriques (Augris et al., 2004) et sur l'évaluation théorique de la mise en mouvement des sables à l'aide de formules semi-empiriques de transport.

\section{Le secteur d'étude}

\section{Des falaises à recul rapide}

4 Le littoral du Pays de Caux (fig. 1), qui borde la Manche orientale sur $130 \mathrm{~km}$ de long, depuis la baie de Seine au sud-ouest jusqu'aux abords de la baie de Somme au nord-est, se caractérise par des falaises crayeuses, plus ou moins riches en lits de silex, d'altitude moyenne de $70 \mathrm{~m}$ (photo 1). Ces falaises, dont le recul moyen actuel est de l'ordre de 20 $\mathrm{cm} /$ an (Costa et al., 2001), sont localement interrompues par des vallées suspendues (valleuses) ou de profondes vallées drainées dans lesquelles se sont implantées les populations et leurs activités.

Figure 1 : Carte de localisation 


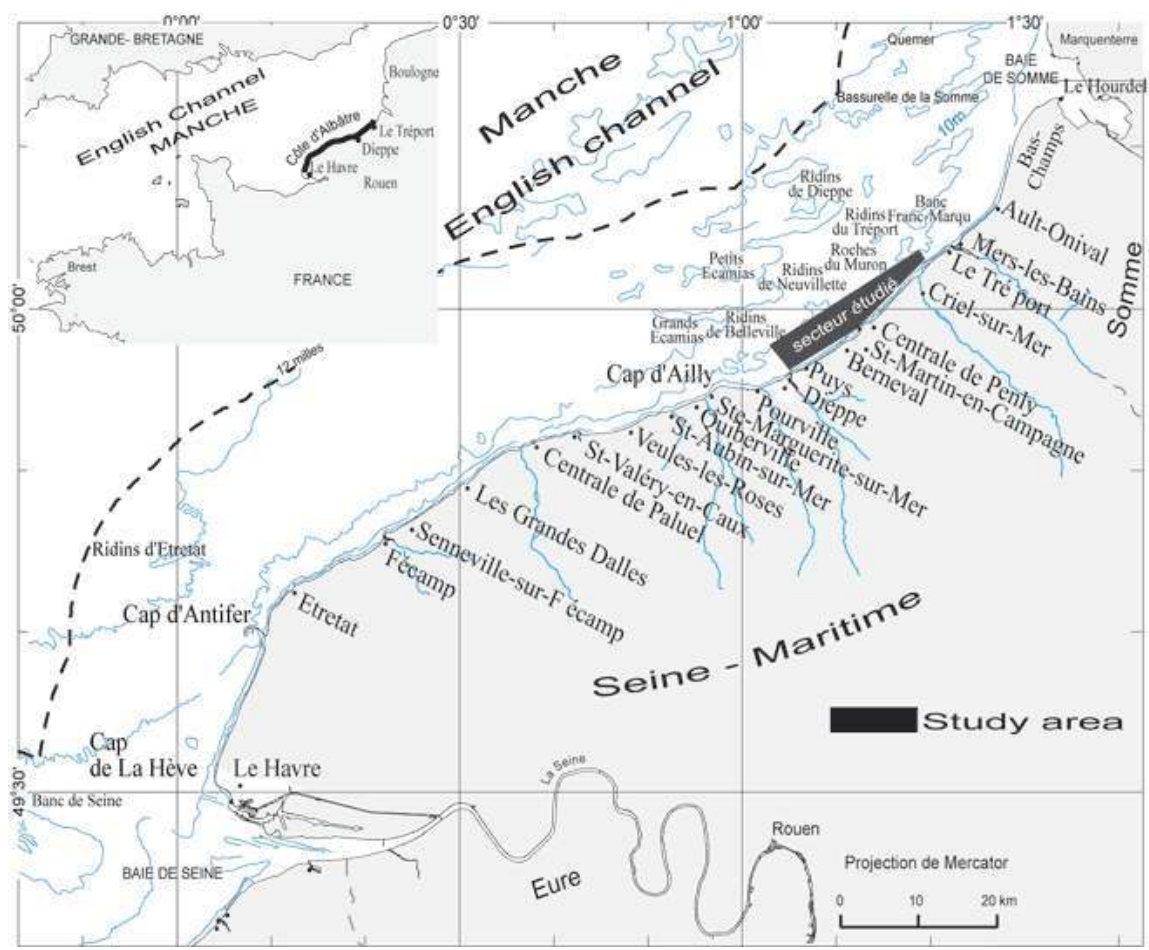

Photo 1 : Le système falaise crayeuse/plate forme/cordon de galets haut-normand (cl. S. Costa) The system of chalk cliff/shore plateform/gravel beach of Haute-Normandy

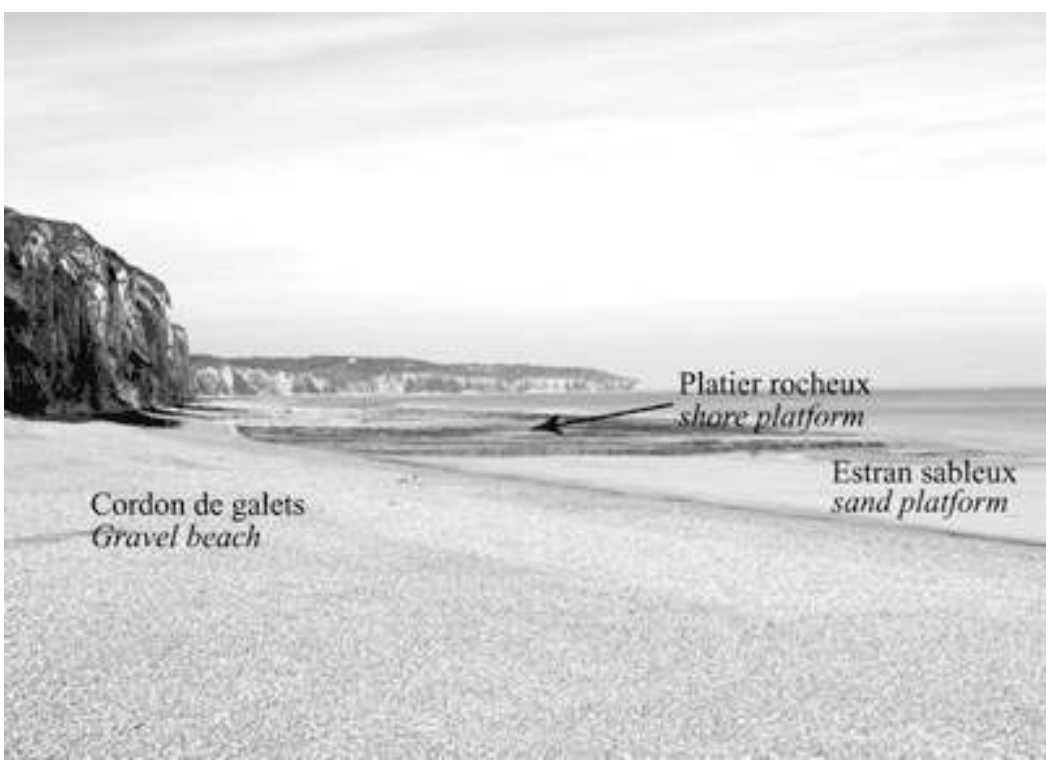

5 Ce littoral correspond à la terminaison nord-occidentale du bassin sédimentaire de Paris qui se prolonge vers l'ouest (en Seine-Maritime) par le plateau du Pays de Caux presque essentiellement composé par les assises crayeuses du Crétacé supérieur. 


\section{Les formes d'accumulations de l'estran}

6 Au pied des falaises, se développe une plate-forme d'érosion marine de largeur comprise entre 150 et $250 \mathrm{~m}$. Ce platier rocheux est masqué dans sa partie supérieure par un cordon de galets de silex alimenté par l'érosion actuelle des falaises.

7 Les cordons de galets adossés aux abrupts crayeux ont une largeur faible ( $15 \mathrm{à} 20 \mathrm{~m}$ ) et une épaisseur d'environ 2 à $2,5 \mathrm{~m}$. En revanche, ceux qui barrent le débouché des vallées ont une largeur moyenne de $40 \mathrm{~m}$, pouvant atteindre 50 à $60 \mathrm{~m}$, et un volume plus important en raison de la présence d'un ouvrage transversal (épi ou jetée) qui bloque leur transit. Les caractéristiques granulométriques de surface des cordons de galets sont relativement simples et assez homogènes sur l'ensemble du secteur d'étude (LCHF, 1972 ; Costa, 1997). Ainsi, la dimension moyenne des galets est de l'ordre de 4 à $7,5 \mathrm{~cm}$ et ne dépasse que rarement $14 \mathrm{~cm}$.

8 L'estran se caractérise également par la présence d'une fraction sableuse non négligeable apparaissant localement sous la forme de placages sur la plate forme d'érosion marine, ou constituant la structure interne des cordons de galets. Sur le platier rocheux, ces placages sableux sont très mobiles et transitent vers le NE (à l'est du cap d'Antifer) en raison de la dominance des houles d'ouest. Particulièrement nombreux entre Saint-Valéry-en-Caux et Le Tréport, ils ont une extension de quelques centaines de mètres carrés, et une épaisseur atteignant parfois $1,5 \mathrm{~m}$. D'autres, plus stables et plus épais, correspondent au comblement des paléo-chenaux des vallées inscrits dans le platier.

9 Les cordons de galets contiennent une importante fraction sableuse (photo 2). En effet, ces derniers sont constitués, au plus, de $50 \%$ de galets, le reste étant un mélange de graviers et de sables grossiers dans une proportion pouvant atteindre 70 \% (LCHF, 1974; Costa, 1997; Morel, 1997; Dolique, 1998). De plus, lors d'épisodes météo-marins paroxysmaux, le suivi de l'évolution granulométrique des cordons de galets montre qu'ils sont fréquemment recouverts, en milieu et en bas de cordon, plus rarement au sommet, par une fraction sableuse pelliculaire (Costa, 1997, 1999). Ce recouvrement partiel est particulièrement notable en fin de forte agitation puis disparaît rapidement par percolation. 
Photo 2 : Fraction sableuse dans le cordon de galets $(A)$ et le recouvrant après un fort coup de vent (B) (cl. S. Costa)

Sandy Fraction in the gravel beach $(A)$ and over lapping it after a storm (B)

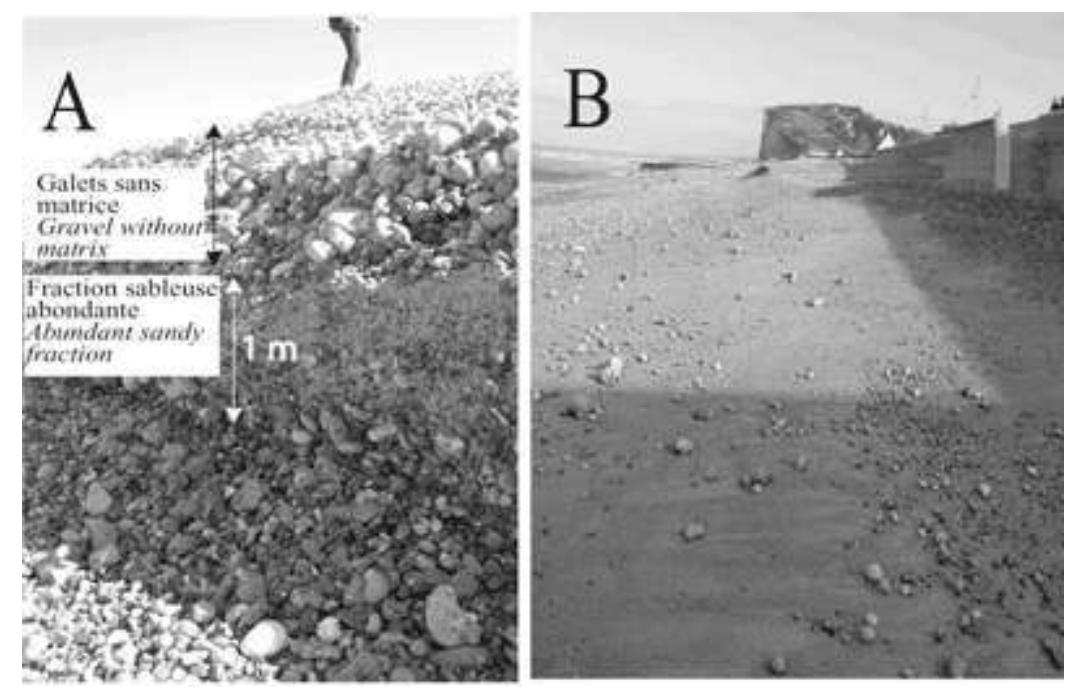

\section{Caractéristiques morpho-sédimentaires des fonds marins}

Les fonds de la Manche orientale sont caractérisés par un relief peu mouvementé et des profondeurs excédant rarement $50 \mathrm{~m}$. Au droit du Pays de Caux, la topographie de la plate forme rocheuse, comprise entre 0 et $20 \mathrm{~m}$, est également peu mouvementée. La pente moyenne est faible, de l'ordre de $0,7 \%$, et croît de la baie d'Authie (0,1 \%) à Antifer (2\%).

11 Les fonds sont recouverts d'une pellicule mince (d'épaisseur métrique) et discontinue de sédiments meubles (sables et cailloutis) (Auffret et Larsonneur, 1975; Reynaud et al., 2003). Ils sont parfois marqués, notamment dans le Pas-de-Calais et la baie de Seine, par des bancs sableux de 10 à $40 \mathrm{~m}$ d'épaisseur. Des formes d'accumulation isolées, appelées « ridins », localisées aux abords de la côte, dans les fonds de 15 à $30 \mathrm{~m}$, se situent au droit du cap d'Antifer et entre Dieppe et la baie de Somme (Auffret et al., 1980; Augris et al., 1993). Ces formations superficielles surmontent un substratum rocheux (calcaire) et se décomposent en une unité inférieure constituée par des sédiments grossiers (cailloutis, graviers, sables grossiers), et une unité supérieure caractérisée par des sables moyens à fins. Ces sédiments sont essentiellement lithoclastiques, et portent les marques d'actions marines et continentales issues de leur histoire anté-holocène en domaine périglaciaire. Redistribués par la transgression flandrienne et progressivement contaminés par des bioclastes, leur répartition est contrôlée par les houles et les gradients de vitesse des courants de marée (Larsonneur, 1971; Aloisi et al., 1977 ; Auffret et al., 1980 ; Anthony, 2002). Ainsi, là où les courants sont les plus forts (entre le Cap d'Antifer et Saint-Valéryen-Caux), les fonds sont caractérisés par l'omniprésence de cailloutis de taille supérieure à $20 \mathrm{~mm}$ (fig. 2). De part et d'autre de ce secteur, la diminution des vitesses des courants de marée induit un gradient d'affinement des sédiments vers les baies de Seine et de Somme. Du large vers les fonds de 6 à $10 \mathrm{~m}$, les matériaux s'affinent également, allant des cailloutis aux sables fins parfois envasés. 
Figure 2 : Relation entre la répartition des sédiments superficiels et la vitesse des courants de marée (Costa, 1997, d'après Larsonneur et al., 1978)

Relation between the location of the superficial sediments and the velocity of tide currents

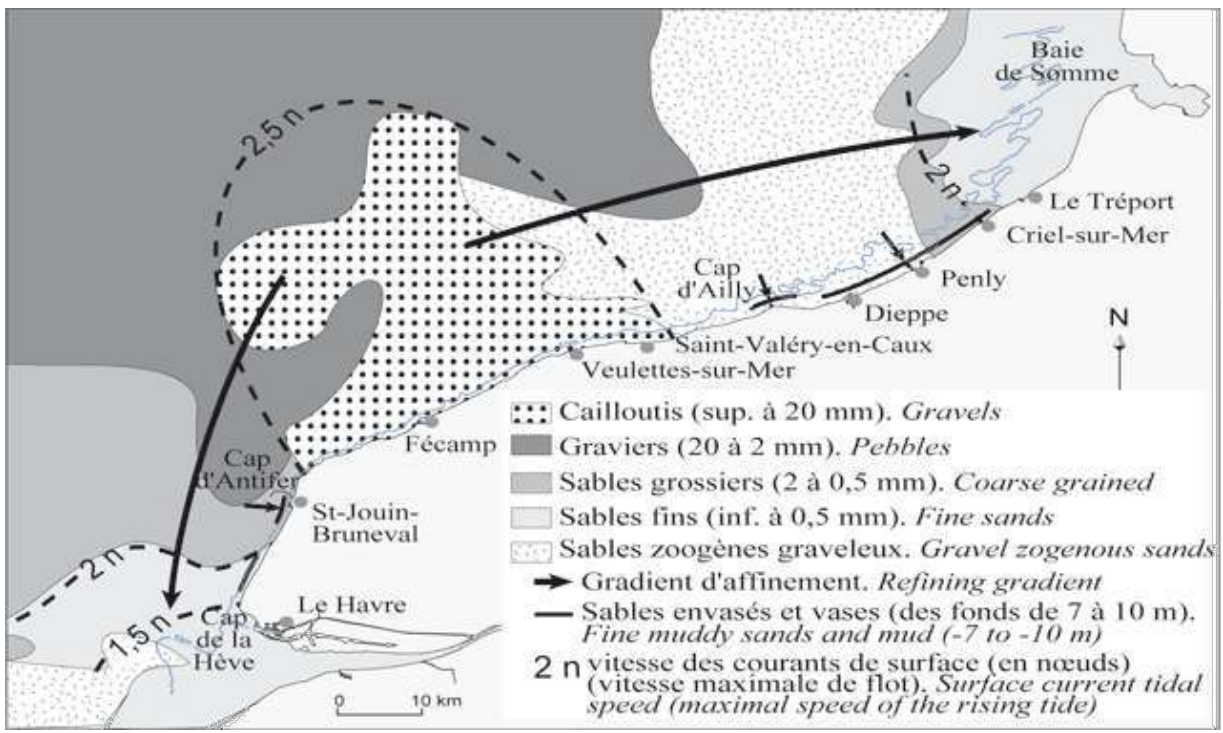

\section{Les conditions hydrodynamiques}

Le littoral haut-normand est un milieu ouvert et exposé, notamment aux houles d'ouest à nord-ouest. En raison des faibles fetchs en Manche, pour la plupart des directions de vent, les agitations sont le plus souvent de type «mer du vent». Les houles les plus fortes mesurées au centre du secteur d'étude (Penly) ont respectivement une hauteur significative annuelle et décennale de 3,8 $\mathrm{m}$ et $4,7 \mathrm{~m}$, et maximale de $4,2 \mathrm{~m}$ et 5,8 $\mathrm{m}$. Leur période moyenne est de 7 à 9 secondes, et leur direction dominante est de secteur ouest (Allen et Delannoy, 1990). Toutefois, la hauteur significative de la houle est dans $90 \%$ des cas inférieure à 1,5 $\mathrm{m}$ et ne dépasse $3 \mathrm{~m}$ que 25 heures.an $^{-1}$ (Teisson, 1986).

La Manche orientale est un environnement de type macrotidal. Les amplitudes de la marée, de cycle semi-diurne, sont de l'ordre de 8,5 m en marée de vive-eau moyenne et croissent du sud vers le nord (SHOM, 1968; LCHF, 1972). Les vitesses maximales des courants en vive eau moyenne croissent du cap de la Hève $\left(0,6 \mathrm{~m} \cdot \mathrm{s}^{-1}\right)$ à Étretat (près de 2 $\mathrm{m} . \mathrm{s}^{-1}$ ), puis décroissent progressivement vers l'est pour atteindre $0,9 \mathrm{~m} . \mathrm{s}^{-1}$ devant la baie de Somme (SHOM, 1999). En morte-eau, ils perdent 20 à $30 \%$ de leur vitesse. Leur direction, qui varie au cours du cycle tidal, est approximativement parallèle au rivage, mais le dessin du littoral ou les forçages météorologiques sont susceptibles de la perturber ponctuellement (Augris et al., 1993 ; Janin et Dumas, 1993 ; Costa, 1997).

\section{L'évolution des petits fonds}

\section{Méthodes et techniques retenues}

Les données sous-marines utilisées proviennent des campagnes réalisées par l'IFREMER en 1988-1989, 1992, 1996, 2000, dans le cadre du suivi écologique de la centrale EDF de Penly. Ces campagnes quadriennales de cartographie morpho-sédimentaire font du secteur Dieppe-Le Tréport le site marin le plus suivi en France. Elles ont permis 
d'observer et d'interpréter les modifications des fonds marins à échéance périodique. L'acquisition des données morpho-sédimentaires est réalisée à l'aide d'un sonar à balayage latéral (110 kHz), et celles-ci sont caractérisées par des prélèvements à la benne. Après numérisation et géoréférencement, au sein d'une système d'information géographique, les données sont analysées dans une perspective diachronique (Beslin et Di Nocera, 1995 ; Gourmelon et al., 1998 ; De La Torre et al., 2002 ; Augris et al., 2004). Deux types de traitement ont alors été opérés sur la zone commune aux quatre levés qui s'étend sur $17,5 \mathrm{~km}$ de long et $3 \mathrm{~km}$ de large, soit $52,5 \mathrm{~km}^{2}$. Au-delà de l'analyse spatiale des données morpho-sédimentaires acquises sur la période récente (1988-1989 à 2000), une comparaison avec une carte bathymétrique plus ancienne du SHOM a été réalisée (1835 et 1989), fournissant des indications quant à l'évolution des fonds.

Afin de confirmer les hypothèses de transferts sableux vers la côte issues de marqueurs morphologiques, diverses formulations semi-empiriques de transport, notamment de début d'entraînement des sédiments ont été utilisées. S'agissant des courants de marée (courants unidirectionnels) la formulation communément adoptée est celle de F. Engelund et E. Hansen (1967) :

$q_{\mathrm{s}}=0,05 \sqrt{\frac{\Delta d_{50}^{3}}{\mathrm{~g}} C_{h}^{2} \tau_{s}^{5 / 2}}$ avec :

$q_{\mathrm{s}}=$ débit solide exprimé en $\mathrm{m}^{3} \cdot \mathrm{s}^{-1} ; \Delta=$ densité apparente du sédiment $: \Delta=\left(\mathrm{P}_{\mathrm{s}}-\mathrm{P}_{\mathrm{e}}\right) / \mathrm{P}_{\mathrm{e}}$

$17 \mathrm{P}_{\mathrm{s}}=$ masse volumique du sédiment $; \mathrm{P}_{\mathrm{e}}=$ masse volumique de l'eau $; \mathrm{d}_{50}=$ diamètre médian du grain ;

$\mathrm{g}=$ accélération de la pesanteur $; \mathrm{C}_{\mathrm{h}}=$ coefficient de frottement sur le fond de Chézy ; 酒造 $*$ = contrainte adimensionnelle globale de cisaillement sur le fond.

19 Concernant l'action de la houle (courants oscillatoires), la formule de R. Soulsby (1997) a été choisie :

$q_{\mathrm{s} 1 / 2}=5,1\left[\mathrm{~g} \Delta d^{3}\right]^{1 / 2}\left(\theta_{h}-\theta_{c r}\right)^{3 / 2}$

avec $q_{\mathrm{s} / 2}=$ débit solide pendant un demi-cycle de houle (c'est-à-dire celui portant vers la côte ou vers le large); $\theta_{h}=$ contrainte adimensionnelle sur le fond; $\theta_{c r}=$ contrainte adimensionnclle critique de début d'entraînement, obtenue à partir de la vitesse orbitale critique sur le fond.

20 Par ailleurs, afin de définir les facteurs responsables de l'évolution morphosédimentaire des fonds observés, une recherche de corrélation entre les conditions de vent à Dieppe et les modifications morpho-sédimentaires a été menée à partir des séries chronologiques de vents trihoraires de 1985 à 1997 (Augris et al., 2004). L'analyse ne porte pas directement sur les valeurs aérologiques enregistrées, mais sur l'énergie annuelle globale des vents de mer (SO à NE), évaluée par le cumul des carrés des vitesses de vent, pondérés par la fréquence d'occurrence. De même, la composante conduisant à une dérive littorale vers le $\mathrm{NE}$ et vers le SO ont été évaluées.

\section{Évolution morpho-sédimentaire du domaine marin entre 1988-1989 et 2000}

21 Conformément à ce qui est observé en Manche, les fonds marins entre Dieppe et Le Tréport sont composés, en 1988-1989, d'un substratum crayeux affleurant localement 
sous forme de petits platiers, qui n'occupent que $3 \%$ des fonds (fig. 3). La roche est généralement masquée par une couverture sédimentaire meuble peu épaisse, comportant des sables fins à moyens ( $59 \%$ de la surface totale), surmontant des sédiments grossiers (sables grossiers aux cailloutis, $33 \%$ ). Les sables fins envasés, localisés dans les petits fonds des abords de Penly, n'occupent que $4 \%$ des fonds. Morphologiquement, les sables se caractérisent en 1988-1989 par le très faible développement de formes sédimentaire transversales au courant, de hauteur comprise entre $5 \mathrm{~cm}$ et $1,5 \mathrm{~m}$ et de longueur d'onde variant de 2 à 20 mètres, appelées mégarides, qui ne représentent qu'environ $1 \%$ des fonds.

Figure 3 : Répartition des formations superficielles en 1988-1989 (Augris et al., 1993) Location of the bed superficial deposits in 1988-1989

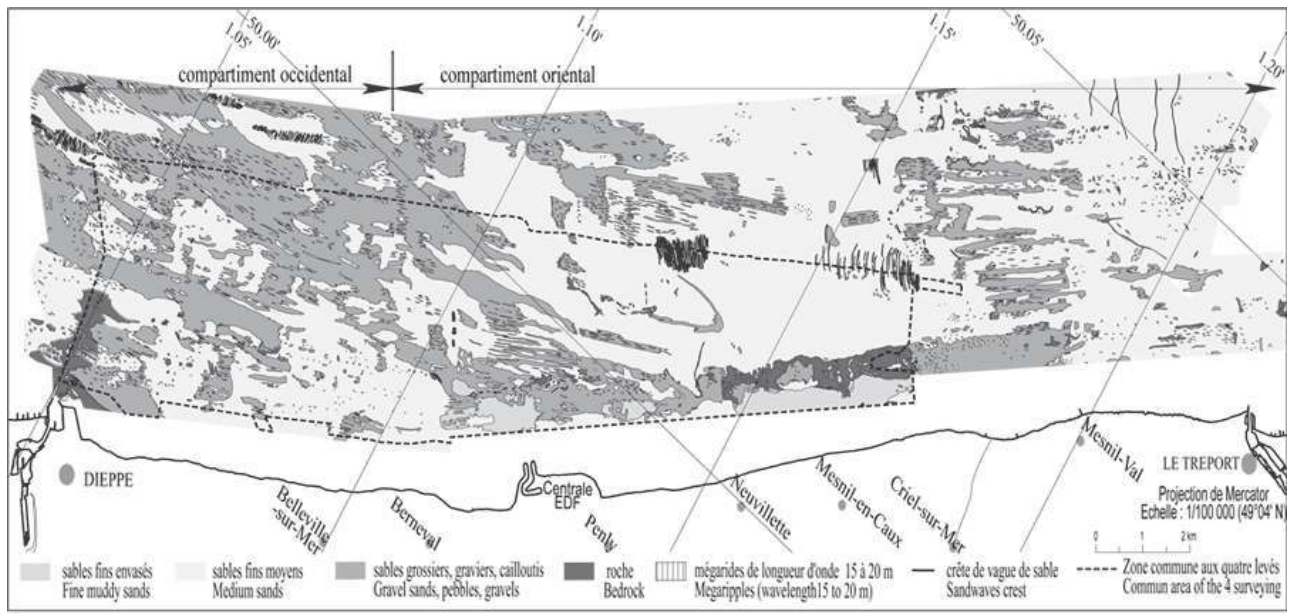

La répartition spatiale de ces types de sédiments permet de définir deux compartiments distincts situés de part et d'autre de Penly :

- dans le compartiment occidental, les fonds sableux et grossiers occupent des superficies pratiquement identiques, respectivement $51,5 \%$ et $46 \%$. Les sables sont localisés dans les zones en " creux ", sous la forme d'un enchaînement complexe de rubans et de taches sableuses, allongées dans le sens ouest-est, obliques de $15^{\circ}$ par rapport à la côte, et dont la surface est modelée par des mégarides de longueur d'onde de 15 à $20 \mathrm{~m}$. Ces formes témoignent d'un faible volume de sable disponible (Belderson et al., 1982). La roche affleure sur $2 \%$ des fonds, principalement devant Dieppe, sous la forme d'un platier. Les sables fins envasés ne constituent que $0,2 \%$ de la surface totale.

- dans le compartiment oriental, les sables moyens à fins dominent largement et constituent une couverture plus continue, et probablement plus épaisse, permettant la formation localisée de figures sédimentaires transversales de type mégaride et vague de sable. Ils occupent près de $70 \%$ de la superficie, alors que les sédiments grossiers en occupent moins de $19 \%$. Parallèlement à leur soudure progressive, l'orientation des figures sédimentaires se modifie progressivement pour devenir parallèle à la côte. La roche occupe moins de $4 \%$ des fonds, et les sables fins envasés sont nettement plus abondants que dans le secteur oriental, avec $7,5 \%$ de la surface. Ils s'organisent parallèlement à la côte à des profondeurs comprises entre 6 et $10 \mathrm{~m}$.

Les évolutions constatées entre 1988-1989 et 2000 concernent principalement le développement de la couverture sableuse (fig. 4). Celle-ci passe de $59 \%$ en 1988-1989 à $72 \%$ en 2000, soit de 31 à $38 \mathrm{~km}^{2}$. L'extension de ce faciès n'est pas régulière au cours du 
temps. Elle est surtout importante entre 1988 et 1992 (de $59 \%$ à 69\%), beaucoup plus limitée ensuite. Elle se produit principalement dans le compartiment oriental, alors que le compartiment occidental ne présente que de légères modifications du système de rubans sableux, sans modification de son orientation générale.

Figure 4 : Variations de l'extension des types de fond de 1988 à 2000 dans la zone commune au quatre levés

Variation of the type of sea floor extension from 1988 to 2000 in the common area of the four surveys

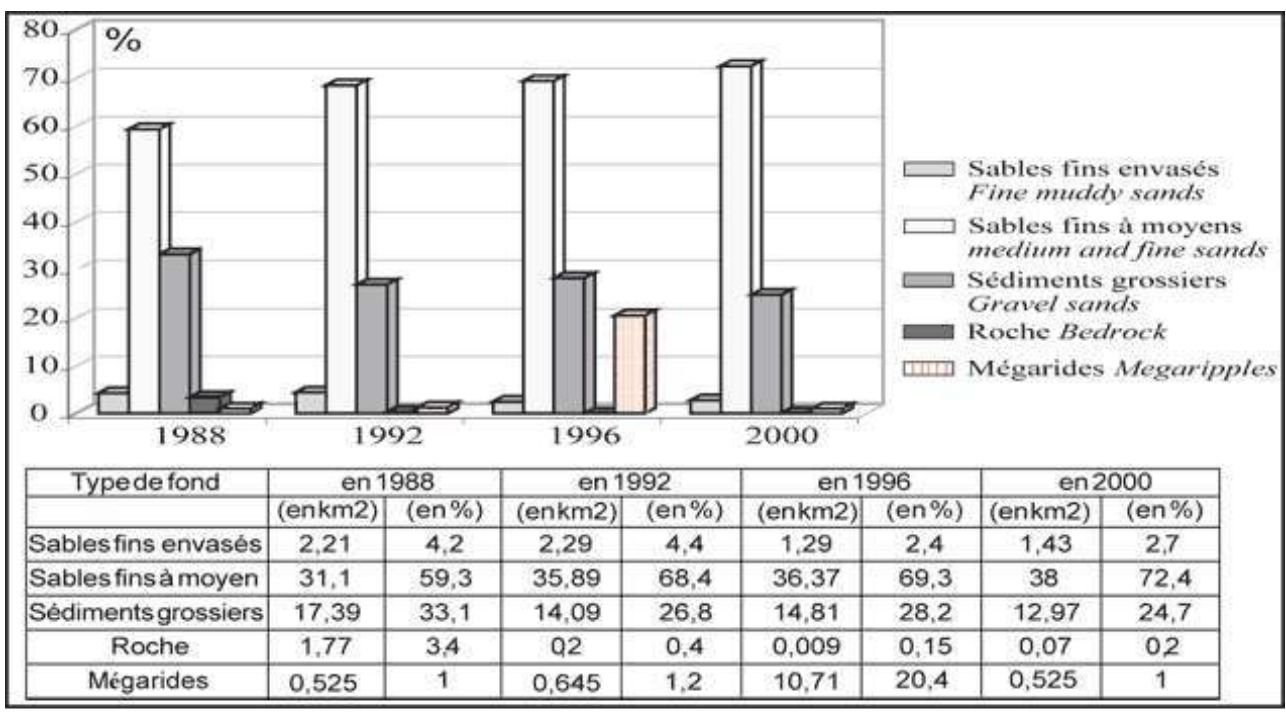

24 Par ailleurs, l'épaisseur de sable mobilisable semble également fluctuer au cours du temps. Les mégarides peu développées en 1988 et en 1992 ( 1 \% de la superficie de la zone), montrent un fort développement en 1996 (20\%, soit plus de $10 \mathrm{~km}^{2}$ ). Ces formes retrouvent, en 2000, des fréquences comparables à celles de 1988 et de 1992. Par conséquent, seul le levé de 1996 diffère des autres en ce qui concerne l'extension des mégarides et, sans doute, l'épaisseur, l'homogénéité, et le stock de sables en surface.

\section{Évolution bathymétrique du secteur Dieppe/Le Tréport à l'échelle séculaire}

Les cartes anciennes (XVII ${ }^{\mathrm{e}}$ siècle) étant peu utilisables en raison de leur imprécision ou de leur caractère trop local, le levé bathymétrique IFREMER de 1988 a été comparé à celui de 1835 réalisé par le Service Hydrographique et Océanographique de la Marine. Les résultats des comparaisons des sondages concernent essentiellement l'évolution moyenne de la bathymétrie sur les extrémités ouest et est de la zone d'étude, la partie centrale n'étant pas exploitable.

La tendance générale d'évolution est plutôt faible et orientée à l'érosion. S'agissant de la morphologie et la nature des fonds, le comportement des secteurs ouest et est diffère. L'extrémité occidentale, au voisinage de Dieppe, se caractérise par une évolution très limitée puisque l'érosion verticale moyenne est de l'ordre de $40 \mathrm{~cm}$ entre 1835 et 1989 . Ce résultat étant à la limite de la précision des levés (marge d'erreur $\pm 30 \mathrm{~cm}$ ), nous en concluons qu'il s'agit plutôt d'une tendance à la stabilité, en cohérence avec la nature majoritairement grossière, et donc peu mobile, des fonds marins. En revanche, 
l'extrémité orientale, au voisinage du Tréport, manifeste une tendance plus forte à l'érosion (érosion moyenne de $1 \mathrm{~m}$ entre 1835 et 1989).

\section{Une répartition et une évolution des types de fond dépendantes des caractéristiques structurales et de mécanismes hydro- sédimentaires}

Parmi les facteurs contrôlant la répartition des différents faciès sédimentaires sur le secteur d'étude, les caractéristiques lithologiques du substrat rocheux semblent déterminantes. En effet, les secteurs de Penly/Criel et de Mesnil-Val/Le Tréport accusent un dénivelé topographique (fig. 5), interprété comme le résultat d'une érosion différentielle entre la craie turonienne, dépourvue de silex, et celle du Sénonien caractérisée par la présence de nombreux lits susceptibles d'interrompre l'érosion verticale (Augris et al., 1993). Cette variation lithologique peut expliquer la répartition plus continue de la couverture sableuse à l'est de Penly: elle provoque une atténuation des vitesses des courants de marée, au dessus des secteurs légèrement déprimés, favorisant la sédimentation. De même, la présence d'un exhaussement de ce substratum au large de Criel se traduit par la rémanence de fonds sédimentaires plus grossiers, résultant de l'accentuation des contraintes dues à la fois au courant (accélération) et à la houle (effet de la réduction de profondeur).

Figure 5 : Morpho-bathymétrie en 1988-1989 Morpho-bathymetry in 1988-1989

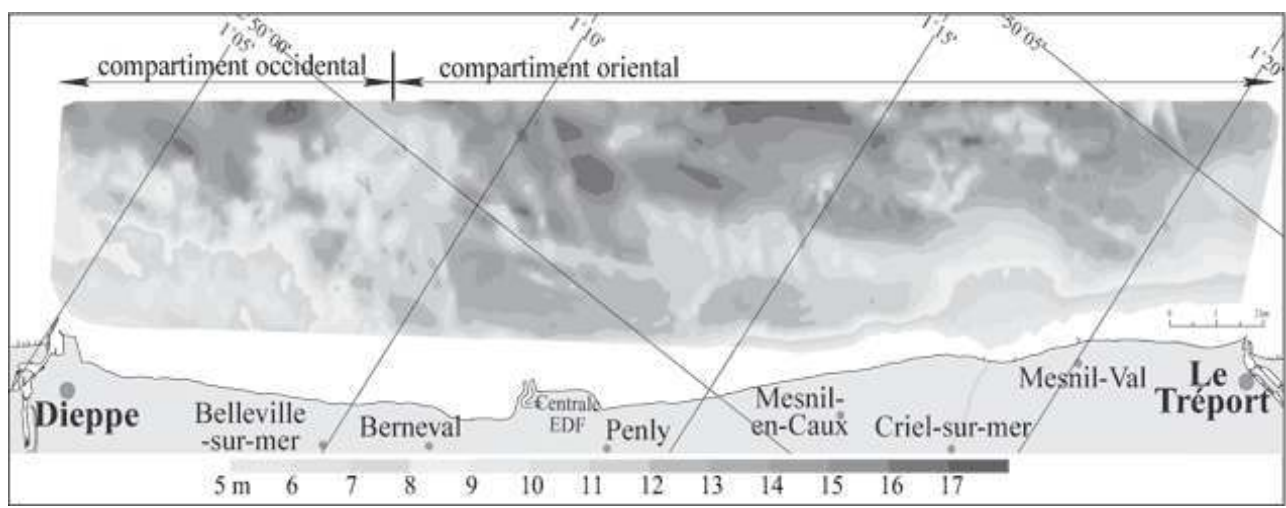

L'analyse sédimentologique des différents faciès fournit également des indications sur les conditions hydrodynamiques locales. Ainsi, la distribution spatiale des sables fins envasés, entre 6 et $10 \mathrm{~m}$, indique une diminution des vitesses des courants de marée vers la côte. Cet espace où l'énergie est minimale correspond à la transition entre la fin de l'influence prépondérante des courants de marée et le début de l'action significative de la houle (Larsonneur, 1971; Auffret et al., 1980 ; Augris et al., 1993). Par ailleurs, la présence, vers $5 \mathrm{~m}(\mathrm{CM})$, de sables fins mais peu envasés suggère qu'à partir de cette profondeur, l'action des houles provoque une remise en suspension ou empêche le dépôt des matériaux les plus fins.

L'examen morphologique des figures sédimentaires (rubans sableux, mégarides, vagues de sables) observées entre Dieppe et Le Tréport met également en évidence deux directions dominantes de transit sur le fond. A l'ouest de Penly, elles indiquent un transit oblique de $15^{\circ}$ par rapport à la côte, induit par le Cap d'Ailly qui modifierait la direction 
des courants de marée. Cette orientation des figures sédimentaires traduirait donc un transit sableux en direction de la côte. A l'est de Penly, conformément à la direction résultante des courants de marée dans ce secteur, les rubans sableux, les marques d'obstacle, les crêtes et l'asymétrie des mégarides et des vagues de sable témoignent d'un transit résultant vers le nord-est, parallèle au rivage.

Dans la partie orientale du secteur d'étude, où les courants sont plus faibles et les épaisseurs sédimentaires en moyenne plus fortes, la tendance à l'érosion semble paradoxale lorsque l'on analyse la répartition des courants. L'atténuation du courant d'ouest en est devrait se traduire par un ralentissement du transport de sédiments, et donc un dépôt. C'est effectivement le scénario qui a permis, notamment lors de la transgression flandrienne et de l'érosion subséquente des fonds sableux de la Manche, la constitution du prisme sableux devant le littoral picard. Mais ensuite, à mesure que la source en sédiments fins se tarit, à l'ouest, les apports aux confins de ce prisme faiblissent par rapport à la capacité de transport (Augris et al., 2004). La limite du prisme sédimentaire sableux semble alors se déplacer progressivement vers l'est (fig. 6), en même temps que les fonds s'érodent.

Figure 6 : Constitution du prisme sédimentaire picard (Auffret et al., 1980) puis progression vers le nord de sa limite septentrionale

Figure 6 : Constitution of picard sedimentary prism, and progression to the North of his septentrional limit

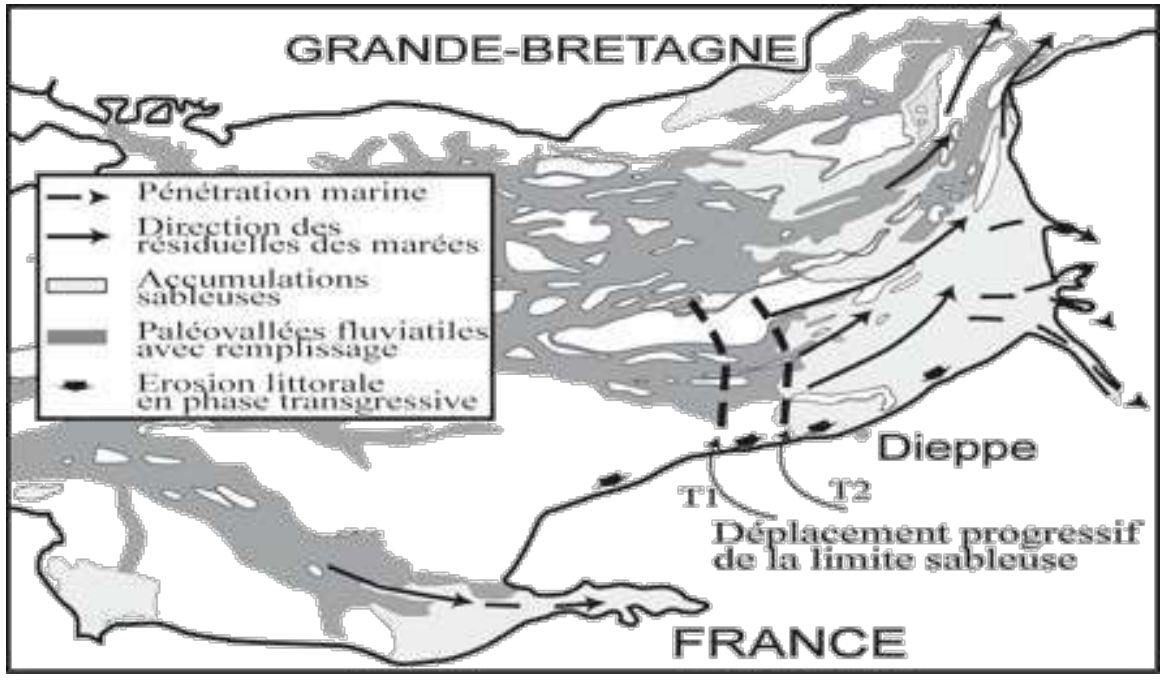

L'évolution morpho-sédimentaire à court terme, particulièrement celle observée entre 1988-1989 et 1996, est remarquable au regard de celle constatée au cours du siècle dernier. En raison de la faible épaisseur des sédiments meubles, il est communément admis que les évolutions significatives à court terme sont souvent étroitement liées aux conditions hydrodynamiques, notamment météorologiques qui influencent les caractéristiques de la houle, ou bien à d'éventuelles modifications des conditions d'apports (ici en provenance de l'ouest). La recherche de corrélation entre l'évolution des fonds, d'une part, et la fréquence et l'intensité des vents forts à Dieppe d'autre part, ne montre pas de relation (fig. 7). Par conséquent, l'extension de la zone à mégarides, et donc l'augmentation du stock de sable mobilisable doivent être liées aux conditions d'apports sédimentaires d'origine externe (apports de sables moyens de l'amont), et/ou interne (migration vers la surface des sables moyens imbriqués dans les sédiments plus grossiers). 
S'agissant de l'augmentation des apports en provenance de l'ouest, il a été évoqué la possible influence des clapages de sables aptiens au large d'Étretat (près de $30 \mathrm{Mm}^{3}$ ), au début des années 1970, lors du creusement du port d'Antifer (Costa, 1997). Toutefois, en raison des volumes nécessaires, ce premier facteur ne semble pouvoir suffire à expliquer, à lui seul, un développement aussi soudain et général du champ de mégarides que celui constaté en 1996. En revanche, il peut être déterminant s'agissant des évolutions constatées dans les très petits fonds et l'estran. Concernant l'ensemble du secteur d'étude, il faut donc faire appel à un autre facteur explicatif. Alors que les apports longitudinaux et transversaux ne peuvent rendre compte de l'évolution des fonds, nous pensons que les fortes tempêtes peuvent mélanger les divers types de sédiments, induisant une migration progressive vers la surface des sables moyens imbriqués dans les formations grossières. Ce classement progressif sur la verticale des sédiments hétérogènes favorise l'apparition d'une couche superficielle de sédiments relativement fins et mieux triés, facilitant alors, le développement graduel de mégarides (Augris et al., 2004).

Figure 7 : Relation entre l'évolution morpho-sédimentaire des fonds et l'énergie des vents de mer à Dieppe entre 1985 et 1997 (Augris et al., 2004)

Relation between the morpho-sedimentary evolution and the sea wind energy at Dieppe between 1985 and 1997

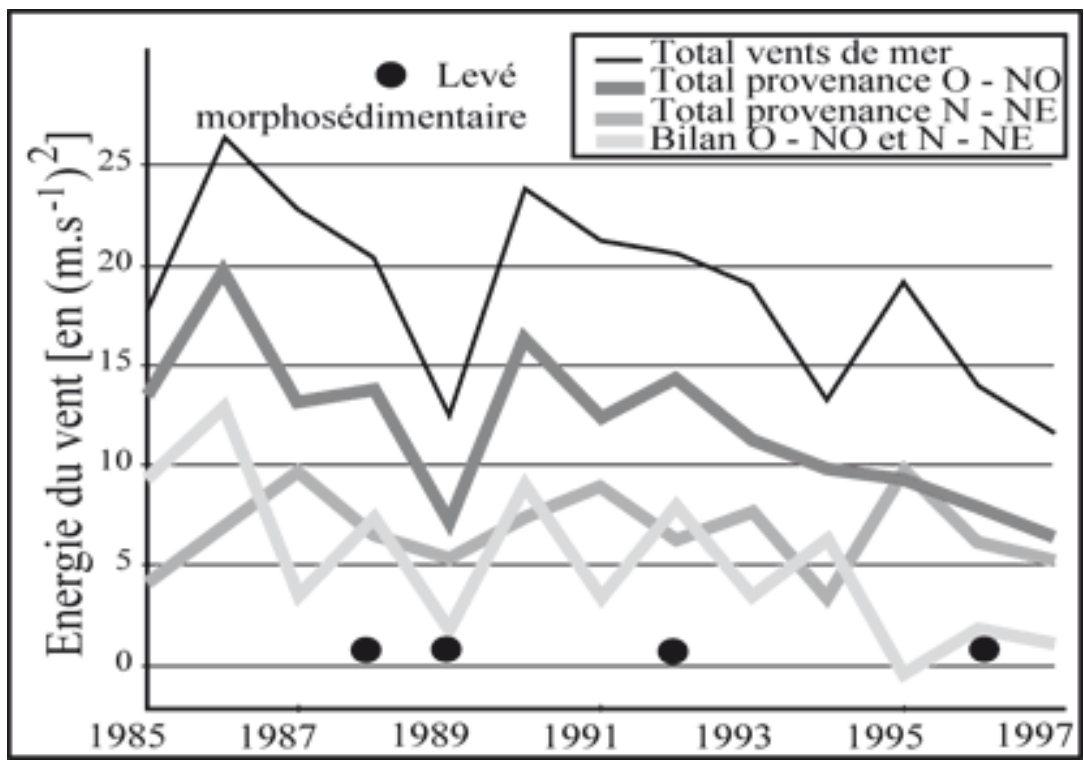

\section{Échanges entre le large et la côte : l'apport de l'approche systémique et pluridisciplinaire}

L'ensablement constaté dans les petits fonds et surtout sur l'estran, au cours des douze années d'observation suggère l'existence d'échanges sableux entre le large et la côte. Toutefois, ces évolutions ont rarement été concomitantes avec les épisodes de fortes houles, traduisant des interrelations complexes et non linéaires, faisant appel à des agents et des processus pouvant se combiner ou se relayer dans le temps et l'espace.

Ce type de fonctionnement, communément admis pour les côtes d'accumulation sableuse, n'est que très rarement envisagé pour les côtes à falaises et à plages graveleuses en raison 
de la prise en compte unique des sédiments grossiers dont la mobilité est quasi exclusivement longitudinale et en haut estran. La mise en évidence de transferts sableux entre le large et la côte pouvant influencer le fonctionnement des plages de galets (Costa, 1997, 1999; Costa et Delahaye, 2002, 2005) implique une approche systémique, et nécessairement pluridisciplinaire. La méthode retenue a consisté à déterminer, à partir de formules de transport semi-empiriques la limite externe du système (la profondeur limite de remaniement des sédiments), et les conditions hydrodynamiques à partir desquelles les sables sont mis en mouvement vers la côte. Cette approche doit permettre, in fine, de proposer un schéma général d'évolution, parfois appelé modèle conceptuel, du système complexe étudié.

L'hypothèse d'une origine infratidale des sables a été privilégiée car les vitesses de recul des falaises et les volumes restreints de sables en présence, dans ou sur ces abrupts, ne peuvent rendre compte des ensablements constatés. De même, une origine fluviatile contemporaine est difficilement envisageable car la charge solide actuelle des fleuves n'est constituée que de matières en suspension argilo-silto-sableuses, ne représentant qu'un volume moyen annuel de $500.10^{3}$ tonnes. $\mathrm{an}^{-1}$ pour la source majeure qu'est la Seine (Avoine, 1986).

\section{Conditions de début d'entraînement des sédiments sous l'action des houles et des courants de marée}

$\mathrm{Vu}$ l'intensité modérée des courants de marée enregistrés sur l'ensemble du secteur d'étude (généralement inférieur à $1 \mathrm{~m} . \mathrm{s}-1$, sauf à l'ouest en période de vive-eau exceptionnelle), et d'après la formulation de F. Engelund et E. Hansen (1967), les courants de marée ne peuvent seuls mettre en mouvement des sédiments supérieurs à $2 \mathrm{~mm}$ dans le compartiment oriental, et d'environ $3 \mathrm{~mm}$ dans le compartiment occidental où le courant est plus fort. La décroissance de leur intensité vers la côte réduit encore davantage leur efficacité.

Le début d'entraînement des sédiments sous l'action de la houle indique que des houles significatives de $3 \mathrm{~m}$ (de l'ordre de la hauteur annuelle) et de période de 8 secondes, agissant à pleine mer de vive-eau, ont une action significative sur les fonds limitée à une bande de moins d'un kilomètre, soit au dessus de $-8 \mathrm{~m}$ (Costa, 1997 ; Augris et al., 2004) (fig. 8). En revanche, à basse mer de vive-eau, elle intéresse quasiment toute la zone d'étude avec des vitesses orbitales sur le fond comprises entre 0,5 et $1,3 \mathrm{~m} \cdot \mathrm{s}^{-1}$, c'est-à-dire susceptibles de mettre facilement en mouvement des sables grossiers.

Figure 8 : Champ de vitesse orbitale de houle sur le fond pour une houle de nord-ouest, de $3 \mathrm{~m}$ de hauteur, de période de 8 secondes, et lors d'une basse mer de vive eau (A) et d'une pleine mer de vive eau (B) 
Swell orbital speed field on the sea floor, for a swell from to the NW, height of $3 \mathrm{~m}$, period of 8 secondes, and during a spring low tide $(A)$ and during a spring high tide $(B)$

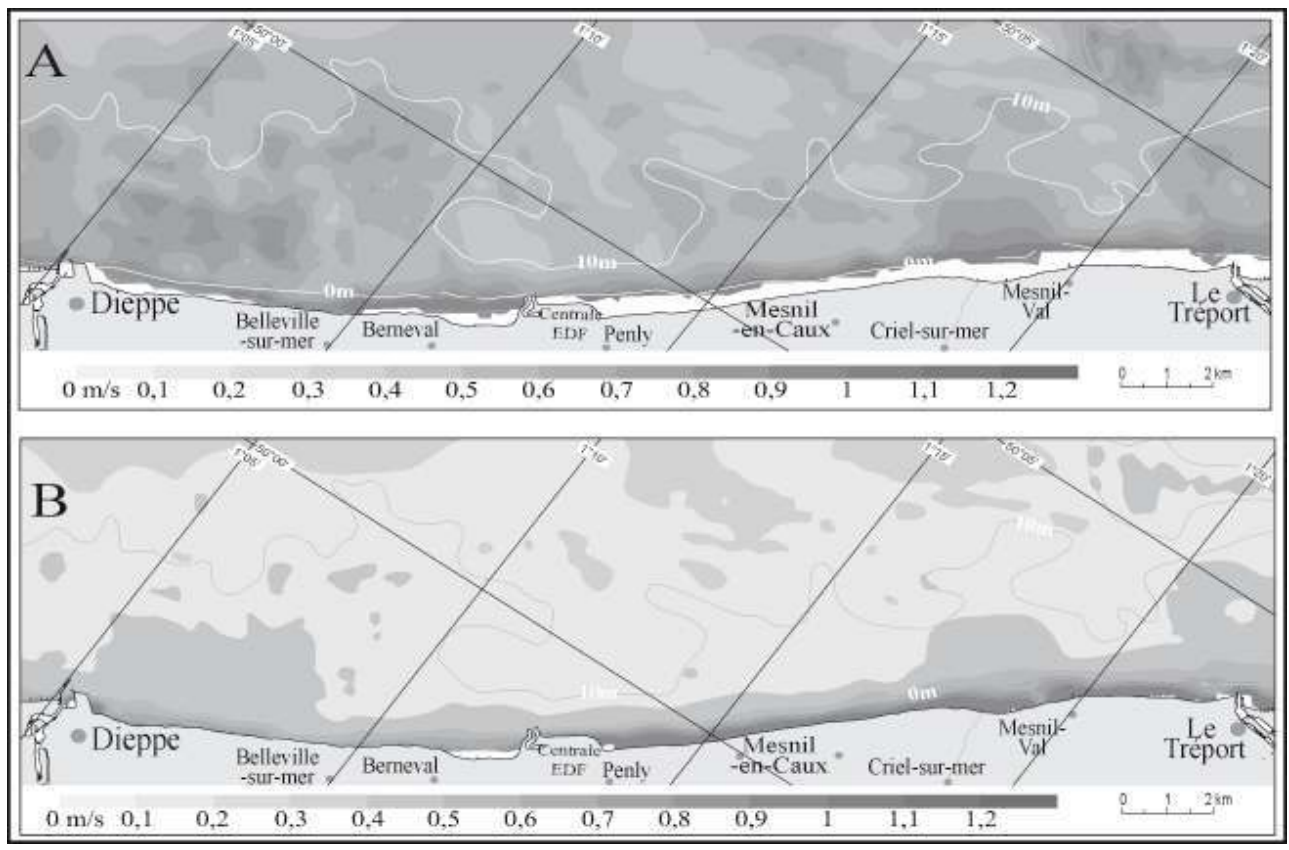

La houle accroît fortement la capacité de transport du courant. De plus, lorsqu'elle est oblique par rapport à ce dernier, elle induit une déviation du transport solide. Ainsi, la houle dominante d'ouest à nord-ouest a-t-elle pour effet de dévier vers la côte le transport sédimentaire dominant.

Même s'il convient de rappeler que les conditions d'agitations permettant un entraînement net des sédiments dans une direction donnée sont plus sévères que celles correspondant au début d'entraînement (oscillation des sédiments), et dépendantes de la pente des fonds, il n'en demeure pas moins que ces résultats confirment que les houles de tempête sont théoriquement capables d'apporter à la côte des sables fins à moyens, lorsque cette agitation survient au moment d'une basse mer de vive-eau (Costa, 1997; Augris et al., 2004). Cette conjonction de facteurs nécessaire à un apport sableux notable vers le rivage rend toutefois ces situations peu fréquentes, mais qui ont été observées en 1992, en particulier sur le site de Penly.

\section{Le cas de l'ensablement massif et soudain de la plage de Penly}

À $10 \mathrm{~km}$ au nord-est de Dieppe, entre les valleuses de Berneval et de Penly, s'est développée une accumulation sableuse qui constitue, avec celles de Quiberville/SainteMarguerite-sur-Mer et de Saint-Aubin-sur-Mer, l'une des plages de sable les plus importantes du littoral de Seine Maritime (photo 3). Cette sédimentation récente et locale apparait bien singulière dans le paysage littoral local par sa situation sur un platier rocheux, sa pérennité et son volume. 
Photo 3 : La plage de Penly délimitée au Nord par la jetée de la centrale EDF (cl. S. Costa) The beach of Penly delimited to the North by the EDF station jetty

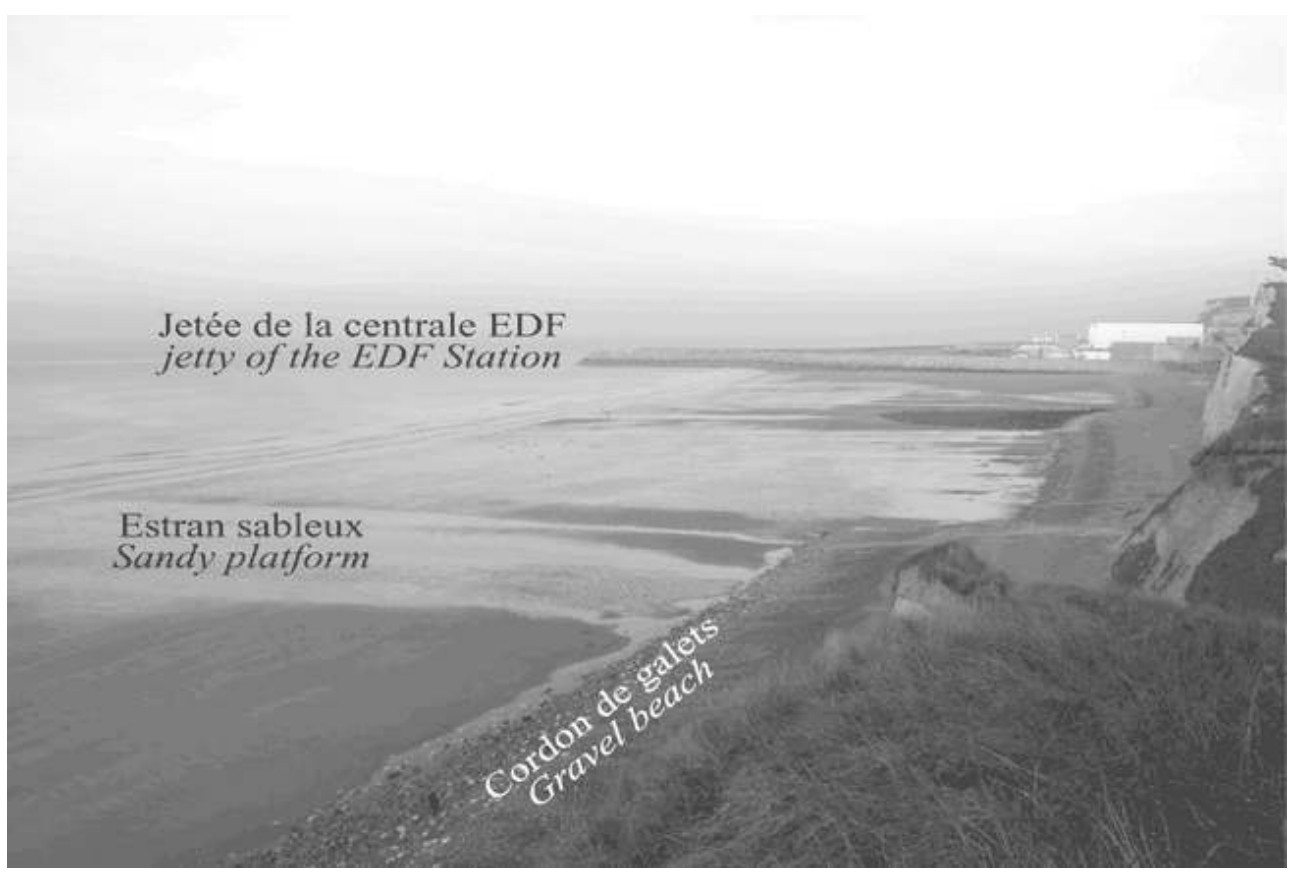

Entre 1981 et 1993, sur une superficie d'environ $0,75 \mathrm{~km}^{2}$, près de $300000 \mathrm{~m}^{3}$ de sable se sont accumulés contre la jetée ouest de la centrale EDF de Penly (implantée en 1981), soit un apport moyen annuel de $25000 \mathrm{~m}^{3}$. Cette sédimentation massive et ponctuelle peut s'expliquer par divers facteurs naturels et anthropiques qui se combinent et se relaient dans le temps et l'espace (Costa, 1997) (fig. 9) :

- une morphologie côtière et des actions anthropiques locales favorables. En effet, entre Berneval et Penly, la confluence de trois valleuses forme un léger rentrant par rapport aux falaises environnantes. Localement, les conditions hydrodynamiques sont atténuées favorisant une sédimentation préférentielle par temps calme. Par ailleurs, en 1981, la construction des jetées du chenal de prise d'eau de la centrale de Penly (500 m de long), au nord-est de cet espace de moindre énergie, a rompu le transit d'estran et accentué la superficie de la « zone d'abri ». De plus, à l'extrémité de l'ouvrage, et à l'ouest du chenal se forme un courant de retour (vortex, matérialisé par les sédiments remis en suspension) lors du jusant ou des houles de secteur nord, accentuant la sédimentation. 
Figure 9 : Origine de l'accumulation de sable contre la jetée ouest de la Centrale EDF de Penly Origin of the sandy deposition against the EDF station occidental jetty of Penly

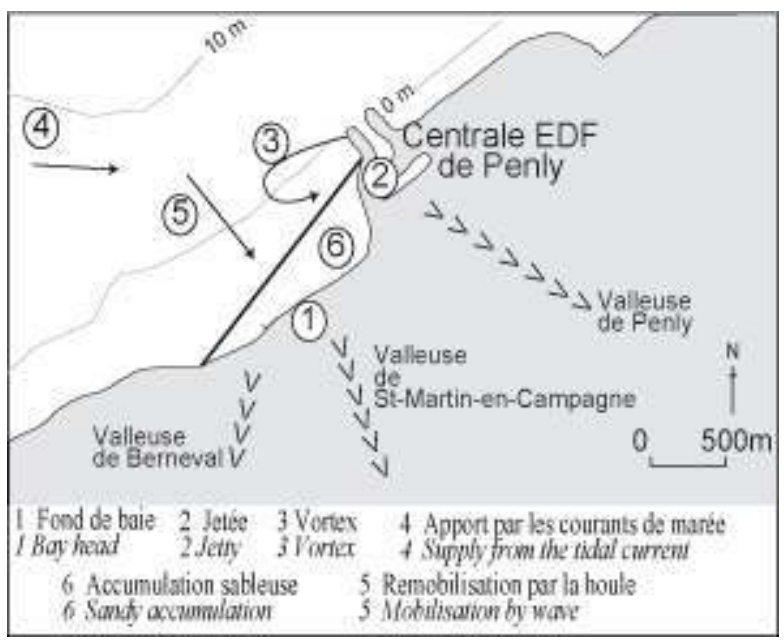

42 Si cet ouvrage transversal constitue l'une des causes essentielles de cet engraissement rapide, il est difficile d'admettre que la seule accumulation des petits placages sableux transitant sur le platier puisse rendre compte de l'ampleur des volumes impliqués sur cette plage. Ils sont d'ailleurs très supérieurs à ceux connus sur la face occidentale de jetées portuaires telles que celles des ports de Dieppe et du Tréport construites au début $\mathrm{du}$ siècle dernier.

- Influence, à plus petite échelle, de la morphologie côtière, et de facteurs hydrodynamiques agissant à divers pas de temps. Il a été démontré que l'irrégularité du trait de côte, notamment le cap d'Ailly, induit localement une déviation des courants de marée, responsable de l'obliquité $\left(15^{\circ}\right)$ des formes d'accumulation sableuse entre Dieppe et Penly (Augris et al., 1993). Cette obliquité indique que la direction de propagation des volumes sableux sous l'action des courants de marée est orientée, ici, vers la côte, et permet à d'importants volumes sableux de se rapprocher du rivage. Toutefois, la décroissance rapide des courants tidaux à l'approche des très petits fonds $\left(0,4 \mathrm{~m} \cdot \mathrm{s}^{-1}\right.$ vers $\left.-6 \mathrm{~m}\right)$, et donc la réduction de leur compétence, ne leur permettent pas d'amener directement cette fraction sableuse sur l'estran. Seules les houles, notamment celles de tempête agissant à basse mer de vive-eau, ont l'efficacité suffisante. Elles relaient donc les courants de marée en permettant, directement, ou selon divers relais spatiaux et temporels, ce transfert des sables depuis les petits fonds jusqu'à la zone intertidale. Ce fonctionnement du système, non linéaire dans le temps et l'espace, semble se vérifier puisque l'ensablement constaté sur la plage de Penly a été particulièrement rapide et intense en 1991-1992, alors qu'aucun fort coup de vent n'a été observé. La seule période pendant laquelle toutes les conditions hydrodynamiques ont été réunies (forte houle agissant à marée basse de vive-eau) est celle des tempêtes de février 1990, soit un à deux ans avant l'ensablement intertidal.

Le système étudié semble fonctionner de la façon suivante. Les courants de marée, déviés localement par le cap d'Ailly, favorisent continuellement et localement le rapprochement des formes d'accumulations sableuses sous-marines près de la côte, qui sont reprises et entrainées épisodiquement dans les très petits fonds par les fortes houles de tempête agissant à basse mer. C'est ensuite sous l'action des houles de tempête plus fréquentes, et peut être certaines houles de beau temps, que les sables seront acheminés sur l'estran. La dérive littorale quotidienne se chargera de les disperser. Les mesures réalisées en mars 
2005 des volumes accumulés sur la plage de Penly indiquent qu'ils sont comparables à ceux estimés en $1993\left(320000 \mathrm{~m}^{3}\right)$. L'explication de cette stabilité réside probablement dans la faiblesse, d'une part, des apports sableux en raison de la rareté des événements tempétueux agissant à basse mer de vive-eau depuis une décennie, et d'autre part, des sorties du système du fait du blocage de la dérive littorale d'estran par la jetée de la centrale EDF de Penly.

\section{Conclusion} importante, la compréhension de l'évolution des fonds et des processus qui en sont responsables reste délicate. Cependant, il s'avère qu'au cours du dernier siècle, la tendance est à l'érosion des fonds sableux au droit du secteur d'étude. Cette érosion semble correspondre à la migration, d'ouest en est, de la limite méridionale du prisme sédimentaire sableux qui s'est constitué au droit du littoral picard et du Boulonnais au cours de la transgression Holocène. Au regard de cette évolution il apparaît donc que la section côtière étudiée constitue une zone charnière, entre un espace de transit des sables (à l'ouest du secteur d'étude) et un espace de sédimentation (à l'est) où s'amorcent les grands bancs de la Manche Est et du Pas-de-Calais.

outefois, cette évolution générale à long terme est ponctuée par des changements significatifs de l'extension des surfaces sableuses sur de courtes périodes (quatre ans). Ces rythmes courts d'évolution, qui ne modifient que très ponctuellement, dans le temps et l'espace, la tendance sur les pas de temps longs, semblent résulter des conditions d'agitations, notamment tempétueuses, qui permettent la migration vers la surface des sables moyens imbriqués dans les sédiments grossiers (Augris et al., 2004).

S'agissant des côtes d'accumulation, notamment sableuses, de nombreuses recherches ont montré l'existence d'interactions complexes entre les plages aérienne et sous marine, qui conditionnent l'équilibre dynamique du rivage. La présente étude montre que cette approche peut également être appliquée pour les côtes à galets de Haute-Normandie. Ces observations sont riches de conséquences puisque ces apports de sables à la côte sont susceptibles d'influencer le volume, la porosité et donc l'aspect réflectif ou dissipatif des plages de galets (Costa, 1997, 1999 ; Costa et Delahaye, 2002, 2005), et qu'ils sont en bonne part à l'origine des processus d'ensablement portuaire. Ces résultats suggèrent également, d'une part, qu'il convient de repousser vers le large les limites du système falaise/platier rocheux/cordon de galets, et d'autre part, que les tempêtes peuvent avoir un rôle ambivalent à différentes échelles de temps. En effet, ces événements morphogènes ont une action fortement érosive sur l'espace intertidal à court terme, mais également un rôle déterminant, à moyen terme, sur l'équilibre sédimentaire des plages par l'apport de sédiments sableux issus des petits fonds et de l'érosion du trait de côte. Cependant, plus que les conditions d'agitation, c'est le budget sédimentaire des estrans et des petits fonds qui conditionne l'équilibre dynamique du système. S'agissant des plages de galets, la présence de sable peut effectivement modifier les caractéristiques de la houle au déferlement, mais cette fraction granulométrique ne constitue qu'un élément mineur dans l'atténuation du risque de submersion de tempêtes des côtes basses car elle ne peut compenser, à elle seule, le déficit chronique en galets. 
La mise en évidence, pour les côtes à galets haut-normandes, d'interactions entre les plages sous-marine et subaérienne, variables dans le temps et l'espace, n'a pu être possible que par l'utilisation de méthodes et d'outils de diverses disciplines. Outre la meilleure compréhension du fonctionnement du milieu, l'approche systémique et par emboîtement d'échelles spatiales et temporelles permet d'apporter des éléments de réponse pour la gestion du littoral, tels que les rejets de dragages, l'envasement des ports, ou encore l'érosion côtière.

\section{BIBLIOGRAPHIE}

AlLEN (H.), DELANNOY (B.), 1990. - « Mesure de houle en différents sites du littoral français », Rapport EDF-LNH HE-45/88.03, 532 p., 388 pl.

ALoisï (J.-C.), BARUSSEAU (J.-P.), MONACO (A.), 1977. - « Rôle des facteurs hydrodynamiques dans la sédimentation des plateaux continentaux ", C.R. Acad. Sci., Paris, sér. D, 280, p. 579-582.

ANTHONY (E. J.), 2002. - « Long-term marine bedload segregation, and sandy versus gravelly Holocene shorelines in the eastern English Channel », Marine Geology, 187, p. 221-234.

AUfFRET (J.-P.), LARSONNEUR (C.), 1975. - « Le modèle sédimentaire de la Manche orientale », IX Congr. Sédim., Nice, 5, p. 33-39.

AufFRET (J.-P.), ALduC (D.), LARSONNEUR (C.), SMith (A. J.), 1980. - « Cartographie du réseau des paléovallées et de l'épaisseur des formations superficielles meubles de la Manche orientale », Ann. Inst. Océanogr., Paris, 56 (S), p. 21-35.

Augris (C.), CLABAut (P)., BOURILlet (J.F.), DREVES (L.), 1993. - « Carte morpho-sédimentaire du domaine côtier entre Dieppe et Le Tréport (Seine-Maritime) », Notice explicative, EDF/IFREMER/ESTRAN, 48 p.

AUGRis (C.), CLABAUt (P.), COSTA (S.), GOURMELON (F.), LATTEUX (B.), 2004. - Évolution morphosédimentaire du domaine littoral et marin de la Seine-Maritime (France), édition Ifremer/Conseil Général de la Seine-Maritime/EDF, 158 p.

AVoInE (J.), 1986. - « Évaluation des apports fluviatiles dans l'estuaire de la Seine. La baie de Seine ", Actes de Colloques $n^{\circ}$ 4, Brest, édition Ifremer, p. 117-124.

BELDERSON (R. H.), JOHNSON (M. A.), KENYON (N. H.), 1982. - « Bedforms », dans Stride (A. H.) (ed.), Offshore Tidal Sands, Processes, and Deposits, p. 27-57.

BESLIN (H.), DI NOCERA (L.), (1995) - « SIG Dieppe/Le Tréport : première étape dans la constitution d'un SIG en milieu marin côtier », Maîtrise de Géographie, Université de Rouen, 86 p.

CARTER (R. W. G.), ORFORD (J. D.), 1984. - « Coarse clastic barrier beaches : a discussion of the distinctive dynamic and morphosedimentary characteristics ", Mar. Geol., 60, p. 377-389.

CoSTA (s.), 1997. - Dynamique littorale et risques naturels : L'impact des aménagements, des variations du niveau marin et des modifications climatiques entre la Baie de Seine et la Baie de Somme, Thèse de doctorat, Université de Paris I, 376 p. 
-, 1999. - « Sédimentologie des plages de galets des côtes françaises de la Manche : application à la défense contre la mer ", Revue de Géographie de Lyon, vol. 74-1/99, p. 35-43.

COSTA (S.), FREIRÉ-DIAZ (S.), DI-NOCERA (L.), 2001. - « Le littoral haut normand et picard : une gestion concertée ", Annales de Géographie, nº 618, p. 117-135.

COSTA (S.), DelAHAYE (D.), 2002. - Pérennité des plages de galets de l'espace Rives-Manche (BERM), Rapport final, Préfecture de Haute-Normandie, Programme Européen Interreg II, 90 p.

-, 2005. - Plages A Risques/Beaches At Risk (PAR/BAR). Rapport final. Conseil Régional de HauteNormandie, Programme Européen Interreg IIIa, $175 \mathrm{p}$.

DE LA TORRE (Y.), GOURMELON (F.), AUGRIS (C.), 2002. - « Contribution d'une Base d'Information Géographique à la connaissance de l'évolution morpho-sédimentaire des fonds marins côtiers : méthodes et limites ", Revue internationale de Géomatique, vol. 12, n 3/2002, p. 311-324.

DOLIQUE (F.), 1998. - Dynamique morpho-sédimentaire et aménagements induits au sud de la Baie de Somme, Thèse de doctorat, Université du Littoral, Dunkerque, $417 \mathrm{p}$.

ENGELUND (F.), HANSEN (E.), 1967. - A monograph on sediment transport in alluvial streams, $3^{\text {rd }}$ edition, Copenhagen, Technical University of Denmark.

GOURMELON (F.), AUGRIS (C.), DI NOCERA (L.), BESLIN (H.), 1998. - « Un Système d'Information Géographique en milieu marin (Dieppe-Le Tréport, Seine-Maritime) », Norois, n 177, p. 75-79.

HESS (L.), HARRIS(W. H.), 1987. - « Effect of storm energy and shoreline engineering on the sediment budget of a barrier beach, Rock-away, New York », Northeast, Geol. 9, p. 110-115.

HiLl (H. H.), KELLEY (J. T.), BELKNAP (D. F.), DICKSON (S. M.), 2004. - « The effects of storm and stormgenerated currents on the sand beaches in southern Maine », Mar. Geol., 210, p. 149-168.

JANIN (J.-M.), DUMAS (F.), 1993. - « Modélisation fine des dérives lagrangiennes en Manche par un code aux éléments finis ». III journées nationales génie côtier génie civil, Sète, mars 1994, EDFHE-42/93.39A, 18 p.

KIRK, (R. M.), 1980. - « Mixed sand and gravel beaches: morphology, process and sediments », Progress in Physical Geography, 4, p. 189-210.

KOMAR (P. D.), 1983. - « Beach processes and erosion - an introduction », dans KoMAR (P. D.) (ed.), CRC Handbook of Coastal Processes and Erosion, Boca Raton (FL), CRC Press, p. 1-20.

LARSONNEUR (C.), 1971. - Manche centrale et Baie de Seine : géologie du substratum et des dépôts meubles, Thèse, Université de Caen, $394 \mathrm{p}$.

LCHF, 1972. - Étude de la production des galets sur le littoral haut-normand, DDE de la SeineMaritime. $63 \mathrm{p}$.

-, 1974. - Étude du littoral entre Le Havre et Antifer. Étude théorique et interprétation de l'ensemble des résultats, DDE de la Seine-Maritime, $135 \mathrm{p}$.

LEVOY (F.), 1994. - Évolution et fonctionnement hydro-sédimentaire des plages macrotidales : l'exemple de la côte ouest du Cotentin (France), Thèse de doctorat, Université de Caen, 423 p.

MAY (J. P.), TANNER (W. F.), 1973. - « The littoral drift power gradient and shorelines changes », dans COASTES (D. R.) (ed.), Coastal geomorphology, University of New York, p. 43-60.

MOREL (V.), 1997. - De la géomorphologie à la gestion des accumulations de galets littoraux du bassin de la Manche et de ses abords : études de cas (Bretagne, Haute-Normandie, Picardie, Sud Angleterre) et réflexions générales, Thèse de Géographie, Université de Brest, 286 p. 
ORFORD (J. D.), FORBES (D.L.), JENNINGS (S. C.), 2002. - « Organisational controls, typologies and time scales of paraglacial gravel-dominated coastal systems », Geomorphology, n 48, p. 51-85.

REYNAUd (J.-Y.), TESSIER (B.), AUfFRET (J.-P.), BERNE (S.), DE BATIST (M.), MARSSET (T.), WALKER (P.), 2003. "The offshore sedimentary cover of the English Channel and its Western Approaches ", Journal of Quaternary Science, 18, p. 361-371.

SHOM, 1968. - Courants de marée dans la Manche et sur les côtes françaises de l'Atlantique, nº 550.

-, 1999. - Les instructions nautiques en France (côtes nord. De la frontière belge au Casquets), SHOM, 241

p.

SHULMEISTER (J.), KIRK (R. M.), 1993. - « Evolution of a mixed sand and gravel barrier system in north Canterbury, New Zealand, during Holocene sea-level rise and still-stand ", Sedimentary Geology, 87, p. 215-235.

SOULSBY (R.), 1997. - Dynamics of marine sands, HR Wallingford, Thomas Telford Publications.

TEISSON (C.), 1986. - « Houle de projet et durées de tempêtes sur le site de Penly », dans Analyse de la houle naturelle, Rapport $\mathrm{n}^{\circ}$ 2, LNH HE-42/86. $14 \mathrm{p}$.

WRIGHT (L. D.), THOM (B. G.), 1977. - « Coastal depositional landforms a morphodynamic approach », Progress in Physical Geography, n 1, p. 412-459.

\section{RÉSUMÉS}

Quatre levés morpho-sédimentaires, obtenus au moyen du sonar à balayage latéral, ont été réalisés sur le domaine marin côtier Dieppe/Le Tréport (Seine Maritime), entre 1988 et 2000 . Les résultats sont intégrés dans une base d'information géographique qui permet d'observer les changements dans la répartition des faciès sédimentaires. Cette analyse montre un ensablement notable des fonds graveleux à l'est de Penly. L'ensablement concomitant de certains estrans rocheux, alors que les apports continentaux sont négligeables, témoigne d'échanges sableux transversaux entre le large et l'estran. L'approche systémique et pluridisciplinaire adoptée permet de définir les relais de processus qui expliquent ces échanges, connus pour les plages de sables, mais rarement identifiés pour les plages de galets.

In 1988 and 1992, four morpho-sedimentary maps of the coastal area Dieppe/Le Tréport (SeineMaritime, France), were made using side scan sonar data. The results were integrated in a geographic information data base, which allows to observe modifications in the sedimentary facies distribution. This study shows that a significant sand enrichment of the gravely sea floor occured east of Penly. The enrichment in sand of some shore platforms, while terrigeneous sand supply is insignificant, is a positive evidence suggesting that cross-shore transport from the shoreface contributes sands to the intertidal zone. The systemic and pluridisciplinary approach adopted in this study allowed us to define the relays of processes which explain these exchanges, known for sandy beaches, but rarely identified for gravel beaches.

\section{INDEX}

Index géographique : France, Seine-Maritime, Dieppe, Le Tréport, Manche (mer de)

Mots-clés : hydrodynamique, littoral, système complexe, transport sédimentaire

Keywords : coastal, complex system, English Channel, hydrodynamic, sediment transport 


\section{AUTEURS}

STÉPHANE COSTA

Université de Caen Basse-Normandie, GEoPHEN, UMR 6554 LETG - CNRS,

stephane.costa@geo.unicaen.fr

\section{FRANÇOISE GOURMELON}

Institut universitaire de la Mer, GEomer, UMR 6554 LETG - CNRS

\section{CLAUDE AUGRIS}

Géosciences marines - Ifremer 3,

PHILIPPE CLABAUT

Géologie littoral et marine 4,

\section{BERNARD LATTEUX}

Ingénierie portuaire et marine 